\title{
CÂNCER DE PRÓSTATA: UMA BREVE REVISÃo ATUALIZADA
}

\author{
PROSTATE CANCER: A BRIEF UPDATED REVISION
}

\author{
Andrey Biff SARRIS'; Fernando José Leopoldino Fernandes CANDIDO'; Carlos Rory \\ Pucci FILHO'; Rodrigo Luiz STAICHAK'; Allan Catarino Kiska TORRANI'; Bernardo \\ Passos SOBREIRO²
}

1: Acadêmicos de Medicina da Universidade Estadual de Ponta Grossa (UEPG) (Ponta Grossa, PR, Brasil). 2: Médico Urologista, Doutor em Clínica Cirúrgica e Professor Adjunto de Urologia da Universidade Estadual de Ponta Grossa (UEPG) (Ponta Grossa, PR, Brasil).

Autor-correspondente: andreybiff@hotmail.com.

\section{RESUMO:}

A próstata é uma glândula presente em homens, cuja principal função é facilitar a fecundação. O câncer de próstata é o segundo tumor mais comum em homens no Brasil e o segundo em termos de mortalidade por câncer. O objetivo do artigo é realizar uma breve revisão bibliográfica atualizada sobre o câncer de próstata. Foi realizada pesquisa nas bases de dados "PUBMED", "SCIELO" e "LILACS" e selecionados os artigos adequados à temática. Diversos fatores de risco estão ligados ao desenvolvimento do câncer de próstata: etnia negra, idade avançada, histórico familiar positivo para esse tipo de câncer, exposição à testosterona e outros hormônios similares, tabagismo, obesidade, entre outros. O rastreio do câncer prostático é feito, basicamente, através de toque retal e dosagem de antígeno prostático específico (PSA). No entanto, diversas mudanças em suas recomendações foram propostas nos últimos anos, as quais serão revisadas neste artigo. O diagnóstico é feito por meio de biópsia guiada por ultrassonografia transretal, com posterior avaliação histopatológica e classificação de prognóstico com base em alguns achados. A depender do estadio estabelecido, diversas modalidades terapêuticas podem ser empregadas: acompanhamento periódico, radioterapia, cirurgia, hormonioterapia ou quimioterapia. Conhecer informações atualizadas sobre câncer de próstata é fundamental, visto sua prevalência e impacto na população.

Palavras-chave: Neoplasias da Próstata; Antígeno Prostático Específico; Próstata.

\section{ABSTRACT:}

The prostate is an important gland of the male body that facilitates the fecundation process. Prostate cancer is the second most common type of malignant tumor among Brazilian men, and presents the second highest mortality rate among all types of cancer. The objective of this article is to carry out a brief and updated literature review about prostate cancer. The research was performed in the databases "PUBMED", "SCIELO" and "LILACS" and the articles adapted to the theme were selected. Several risk factors have been linked to prostate cancer: black ethnicity, advanced age, positive family history to prostate cancer, excessive exposure to testosterone and similar hormones, smoking, obesity, among others. Digital rectal exam and prostate specific antigen (PSA) test are the screening methods currently recommended. However, these recommendations have been submitted to several changes in the past few years, which will be further reviewed in this article. The diagnosis of prostate cancer can only be made with a prostate biopsy and posterior histopathological analysis. Several therapeutic modalities, such as active surveillance, radiation therapy, 
surgery, hormone therapy and chemotherapy, are available and should be selected according to the clinical stage of the tumor. The knowledge of prostate cancer's updated information is important, since prevalence and its health impact are high.

Key-words: Prostatic Neoplasms; Prostate-Specific Antigen; Prostate.

\section{INTRODUÇÃO}

A próstata é uma glândula única com função secretora presente em homens. O líquido prostático tem $\mathrm{pH}$ básico, sendo importante no processo de alcalinização vaginal e conferindo o aspecto leitoso do sêmen e seu odor característico. Além disso, é possível que ela alcalinize os demais líquidos seminais masculinos, melhorando o desempenho de mobilidade do espermatozoide (HALL, 2011 \& SILVERTHORN, 2010). A próstata fica em posição imediatamente anterior ao reto, justificando o exame de toque retal como uma forma de avaliação prostática. De uma maneira geral, as dimensões da próstata são de $3 \mathrm{~cm}$ de comprimento, $4 \mathrm{~cm}$ de largura e $2 \mathrm{~cm}$ de profundidade antero-posterior - classicamente mencionada como tamanho de uma "noz". O tamanho médio aos 20 anos de idade da próstata é de $20 \mathrm{~g}$ e há um crescimento de 0,4g/ano a partir dos 30 anos (BERMAN et al, 2012).

\section{METODOLOGIA}

Foi realizada consulta à literatura científica através das pesquisas nas bases de dados eletrônicos "PUBMED", "SCIELO" e "LILACS" durante os meses de novembro e dezembro de 2017 e também livros com grande arcabouço teórico e de grande impacto na prática de pesquisa. Nas bases, foram utilizadas os descritores "câncer de próstata" e "neoplasia prostática", assim como suas traduções para a língua inglesa. Foram lidos os primeiros títulos de cada base, sendo lidos os resumos dos títulos considerados adequados à temática. Foram selecionados os artigos e revisões que produziram os conhecimentos com maior número de evidências quanto à temática, no sentido de produzir um texto com as informações mais consolidadas na área.

\section{EPIDEMIOLOGIA}

O câncer de próstata é o quinto mais prevalente mundo em ambos os sexos e o segundo em homens, atrás apenas do câncer de pele. Os países desenvolvidos têm maiores taxas de incidência em relação aos países subdesenvolvidos e como será abordado a frente, 
os países com populações asiáticas possuem as menores taxas (ABOUASSALY et al, 2012 \& DARVES-BORNOZ et al, 2014). No Brasil, o câncer de próstata também é o mais comum, desconsiderando o de pele. O Instituto Nacional do Câncer (INCA) estimou que, no ano de 2016, houve quase 62.000 novos casos desse tipo de tumor, correspondendo a aproximadamente $23 \%$ de todos os cânceres diagnosticados nos homens no país. Em termos comparativos, as regiões Sul e Sudeste têm o maior número de casos, com uma taxa de 90 novos casos para cada 100.000 habitantes (INSTITUTO NACIONAL DO CÂNCER, 2016).

\subsection{Mortalidade}

Em termos de mortalidade, é a segunda causa de morte por câncer. De acordo com o INCA, no ano de 2013, houve 13.772 mortes em razão do câncer de próstata (INSTITUTO NACIONAL DO CÂNCER, 2016). Desde o início da aplicação do PSA (Antígeno Prostático Específico), na década de 1980, como forma de diagnóstico precoce, os números vêm decaindo, especialmente nas faixas etárias mais avançadas. Além do diagnóstico precoce, o tratamento de forma mais incisa tem contribuído muito para esses números. A taxa caiu de 31 mortes a cada 100.000 homens no ano de 1975 para 23 mortes no ano de 2005. De uma maneira geral, a taxa de mortalidade também é maior em países desenvolvidos, especialmente no continente americano (ABOUASSALY et al, 2012 \& DARVES-BORNOZ et al, 2014).

\subsection{Diferenças étnicas}

Diferentes etnias têm incidência diferente do câncer de próstata. Isso obviamente não tem relação apenas genética, mas também com vários outros fatores como meioambiente, estilo de vida e dieta. Negros têm maiores chances de desenvolver câncer de próstata, seguido pelos brancos, latinos, asiáticos e por último, os indígenas. Em comparação com os indivíduos brancos, negros têm 1,6 vezes mais chances de ter câncer de próstata e 2,4 vezes mais chances de morrer por esse tipo de câncer (ABOUASSALY et al, 2012; COOPERBERG et al, 2013 \& DARVES-BORNOZ et al, 2014).

\subsection{Idade}

O câncer de próstata é um clássico tumor de populações mais idosas. Apenas $2 \%$ 
dos casos ocorrem em homens com menos de 50 anos. A média de idade dos pacientes é de 68 anos.

\subsection{Estágio do diagnóstico}

Desde a introdução do teste de PSA como tentativa de diagnóstico precoce, os números dos tumores que se restringem apenas à próstata (não fizeram metástase, ou seja, não se expandiram para outras regiões) aumentaram consideravelmente. Isso reflete muito no melhor prognóstico do paciente, ou seja, na maior propensão à cura (ABOUASSALY et al, 2012; COOPERBERG et al, 2013 \& DARVES-BORNOZ et al, 2014).

\subsection{Fatores de risco}

Fatores de risco são, como o próprio nome diz, fatores que aumentam os riscos de ter determinada doença. Entretanto, tê-los não significa que obrigatoriamente ela se desenvolverá. Entre os fatores de risco, podemos citar:

- Idade: Como visto, indivíduos mais velhos têm maiores chances de desenvolver o câncer de próstata, especialmente acima da quinta década de vida;

- Etnia: Negros são grupo de risco para o desenvolvimento deste tipo de tumor;

- Histórico Familiar: Pessoas que têm histórico familiar de câncer de próstata têm grandes chances de desenvolvê-lo também. Quando um parente de primeiro grau possui esse histórico, o indivíduo tem duas vezes mais chances de evoluir com a neoplasia. Além disso, aparentemente a agressividade do tumor também guarda correlação com esse histórico. Pacientes cujos parentes desenvolveram a doença previamente aos 55 anos possuem uma propensão ainda maior de desenvolver a doença, frequentemente em idades mais precoces do que outros indivíduos;

- Fatores Hormonais: A quantidade de hormônios andrógenos (por exemplo, a testosterona) tem impacto na formação tumoral, principalmente em relação ao envolvimento com a reformulação celular prostática. Níveis elevados de testosterona parecem corroborar com o desenvolvimento da neoplasia. Entretanto, os estudos clínicos (estudos com pessoas) tiveram dificuldades em mostrar isso, especialmente em faixas próximas à normalidade. Um outro hormônio, chamado fator de crescimento semelhante à insulina tipo 1 (IGF-1), em níveis elevados, também parece contribuir com a formação do câncer; 
- Tabagismo: Sabe-se que o tabagismo é fator de risco para inúmeros tipos de câncer e isso não é diferente com o de próstata. $\mathrm{O}$ ato de fumar está envolvido principalmente na agressividade tumoral: tabagistas têm, em média, 30\% mais chances de morrer por câncer de próstata que um não-fumante (ABOUASSALY et al, 2012; COOPERBERG et al, 2013 \& DARVES-BORNOZ et al, 2014);

- Obesidade: A obesidade parece ter relação tanto com a mortalidade quanto com o aparecimento do câncer de próstata. No entanto, o que é mais comprovado através dos estudos clínicos é o maior potencial de fatalidade nesse grupo de risco. Isso parece se dar principalmente por um hormônio chamado de Leptina, encontrado em altas concentrações nos obesos (MANDAIR et al, 2014);

- Consumo de Soja: O consumo de soja parece estar relacionado com um menor risco do desenvolvimento do câncer de próstata. Muitos estudos sustentaram essa teoria enquanto outros não acharam relação (MANDAIR et al, 2014);

- Genética: Mutações nos genes BRCA1 e BRCA2 mostraram ser risco independente para o desenvolvimento do câncer de próstata com chances aumentadas em duas e sete vezes, respectivamente. Além disso, nesses pacientes a agressividade tumoral se mostrou bem superior. Mutações em outra região do DNA, na região 8q24 também mostraram os mesmo resultados (ABOUASSALY et al, 2012; COOPERBERG et al, 2013 \& DARVESBORNOZ et al, 2014);

- Inflamação: Pacientes que desenvolvem prostatites parecem ter maior propensão ao desenvolvimento da neoplasia;

- Outros: O consumo de álcool, de gorduras e de vitamina D em alguns estudos mostrou-se maléfico, mas não foi possível chegar a uma conclusão definitiva. Da mesma forma, o consumo de licopeno, vitamina $E$ e selênio pareceu se mostrar protetor, o que também não foi confirmado por outros estudos (ABOUASSALY et al, 2012; COOPERBERG et al, 2013; DARVES-BORNOZ et al, 2014 \& MANDAIR et al, 2014).

\section{RASTREIO (SCREENING)}

No ano de 2012, o rastreio de câncer de próstata sofreu uma reviravolta. Um órgão americano (USPSTF - United States Preventive Service Task Force) fez uma publicação na qual contraindicava o rastreio do câncer de próstata, pois não havia comprovação de redução de mortalidade (MOYER et al, 2012). Nesse mesmo sentido, a Sociedade Brasileira de Medicina de Família e Comunidade e o Instituto Nacional do Câncer aderiram 
a esse protocolo - ou seja, deveria-se parar de realizar toque retal e PSA em pacientes sem sintomas. Logo em seguida, houve o contra-ataque de outras entidades, demonstrando que o documento do USPSTF cometeu diversos erros na análise para elaboração dessa recomendação (não consideraram a redução de mortalidade de 40\% por câncer de próstata nos EUA, ignoraram pesquisas que mostraram redução de mortalidade em grupos de risco, não tinham oncologistas ou urologistas no time de análise, entre outros) (SOCIEDADE BRASILEIRA DE UROLOGIA, 2015).

Sendo assim, a posição atual das associações (Sociedade Brasileira de Urologia, American Urological Association, European Association of Urology, American Cancer Society, MD Anderson Cancer Center, AC Camargo Cancer Center e Sociedade Brasileira de Oncologia Clínica) é de:

- Realizar toque retal em pacientes a partir dos 40 anos de idade até os 75 anos de idade como parte do exame médico;

- Considerar o uso de PSA entre os 40 e 75 anos (desde que haja possibilidade de sobrevida em 10 anos) em: 1. Pacientes com alto risco para câncer de próstata (negros ou com familiares com câncer de próstata antes dos 65 anos); 2 Pacientes sintomáticos; e 3. Pacientes que queiram realizar o exame.

Depois dos 75 anos, os riscos tornam-se muito pequenos (desde que já realizado algum acompanhamento prévio, obviamente) e mesmo que o câncer apareça, é muito provável que o paciente não vá morrer por causa dele.

Esta nova posição quanto às recomendações para rastreio e as críticas impostas à análise dos demais órgãos foram tão contundentes que, no ano de 2017, a USPSTF voltou atrás e fez as mesmas recomendações supracitadas (SOCIEDADE BRASILEIRA DE UROLOGIA, 2017 \& UNITED STATES PREVENTIVE SERVICE TASK FORCE, 2017).

É válido lembrar que o PSA é um marcador órgão-específico e não câncerespecífico. Ou seja, outros problemas de saúde, como inflamação da próstata, podem alterá-lo.

\subsection{Toque retal}

O toque retal para diagnóstico de câncer de próstata deve ser feito preferencialmente pelo médico urologista. Ao realizar o exame, o médico tenta definir algumas características da próstata: tamanho, consistência, superfície, forma, limites e sensibilidade. Isso permite que ele defina se há algum problema e estabeleça o próximo passo. Em geral, alterações 
no toque retal com PSA maior que $3 \mathrm{ng} / \mathrm{ml}$ já são suficientes para indicação de biópsia (EIFLER, 2014 \& LOEB et al, 2012).

\subsection{Ultrassom, Ressonância Magnética e Biópsia}

O ultrassom via abdominal é o exame mais utilizado como primeira escolha para avaliação prostática. O ultrassom transretal, apesar de fornecer informações mais precisas, acaba sendo realizado apenas quando há necessidade de biópsias. Entretanto, novos estudos têm indicado que a ressonância magnética possui melhores resultados do que os exames ultrassonográficos, ainda que seja pouco utilizada devido ao seu alto custo e baixa disponibilidade (TRABULSI et al, 2012).

\section{DIAGNÓSTICO DO CÂNCER DE PRÓSTATA}

O diagnóstico confirmatório de câncer de próstata é feito a partir da biópsia guiada pelo ultrassom transretal. São retirados 12 "pedaços" da próstata para avaliação. Um médico patologista avalia o tecido (com as células) e define se é aquilo é normal, se é benigno ou se é canceroso. Caso seja câncer, o patologista expressa o laudo na chamada Graduação Histológica do Sistema de Gleason. De maneira simplificada, ele avalia duas áreas mais frequentes do tumor dando um número de 1 a 5 para cada uma delas (sendo o 1 o menos agressivo e o 5 mais agressivo) (LOEB et al, 2012). Ele soma os números dados, o que gera a escala de Gleason (variando, portanto, de 2 a 10). Com base em Gleason, podemos estabelecer:

- Gleason de 2 a 4: Aproximadamente 25\% de chance de o tumor disseminar-se para fora da próstata em anos, afetando a sobrevida;

- Gleason de 5 a 7: Aproximadamente 50\% de chance;

- Gleason de 8 a 10: Aproximadamente 75\% de chance.

\section{ENTENDENDO A HISTOPATOLOGIA}

Basicamente, podemos dividir os cânceres de próstata em dois grupos: os de origem epitelial e os de origem estromal (não-epitelial). Eles são mais comuns na zona externa da glândula, o que facilita e explica o toque retal como método preditivo.

Quanto aos de origem epitelial, podemos falar em adenocarcinoma acinar e não- 
acinar. Entre os adenocarcinomas não-acinares temos o adenocarcinoma ductal, o qual apresenta um pior prognóstico. O tumor de próstata mais agressivo, no entanto, é o tumor de pequenas células. Tumores da bexiga podem também se alojar na próstata, porém com menos frequência (OXLEY, 2014).

\section{MARCADORES TUMORAIS}

Marcadores tumorais são moléculas presentes no tumor, sangue ou outros tipos de tecidos corporais, cujo "aparecimento" pode estar relacionado com o desenvolvimento de neoplasias. Um dos marcadores mais conhecidos já foi mencionado: o PSA. O PSA total é o de maior confiança se comparado com suas frações. As formas de PSA e outro marcadores serão relatados a seguir (CHEETHAM, 2014 \& LOEB et al, 2012).

\subsection{Porcentagem Livre de PSA}

A maioria das moléculas de PSA caminha no sangue ligada a proteínas. Seu uso está indicado para paciente com PSA total entre 4 e $10 \mathrm{ng} / \mathrm{ml}$ com toque retal negativo. Em pacientes com câncer, a tendência é que haja diminuição da porcentagem livre. Estima-se que $56 \%$ dos homens com porcentagem de PSA livre menor que $25 \%$ tenham tumor em comparação com os $8 \%$ dos homens que tinham esse valor acima dos $25 \%$.

\subsection{Velocidade e Densidade de PSA}

A velocidade do PSA, ou seja, o seu aumento ou diminuição ao longo do tempo também auxiliam a determinação da probabilidade de câncer de próstata, como já comentado. A densidade de PSA também parece contribuir, porém mais estudos sobre ela ainda são necessários.

\subsection{PSA Inativo}

Algumas enzimas degradam o PSA sanguíneo. Um anticorpo chamado de $M A b$ 5D3D11 tem a capacidade de se ligar a esse PSA, que está aumentado nos casos de câncer. Alguns estudos tentaram associá-lo para prever o câncer de próstata, porém ainda apresentam resultados insuficientes. 


\subsection{PSA Isoformas}

O PSA não é apenas um tipo de substância. Há um conjunto de tipos de PSA com pequenas diferenças determinada isoformas (mesma forma). A isoforma conhecida por p2PSA tem se mostrado mais especifica e com maior acurácia do que apenas a taxação do PSA total. Outra isoforma é o ProPSA, que é utilizada juntamente com o PSA total e o PSA livre pra mensuração do Índex de Saúde Prostático, o qual parece ter um futuro promissor.

\subsection{Fator de Crescimento Semelhante à Insulina Tipo 1 (IGF-1)}

O IGF-1 tem potente função proliferativa e de inibição de apoptose no corpo humano, especialmente sobre a próstata. Na prática, veem-se níveis elevados em pacientes que desenvolverão câncer de próstata e/ou já o tem, porém com níveis reduzidos nos tumores de estágios avançados.

\subsection{Calicreína Glandular Humana Tipo 2 (hK2)}

A calicreína glandular humana tipo 2 (hK2) é produzida pelo epitélio prostático com concentrações até 100 vezes menores que o PSA. Ademais disso, hK2 é superexpressa nos tumores, sendo especialmente importante para detecção de tumores extracapsulares.

\subsection{Antígeno de Câncer de Próstata 3 (PCA3 - PROGENSA®)}

O gene do PCA3 é específico do tecido prostático e seu RNA (ácido ribonucleico) é superexpresso nos pacientes com câncer. Seus valores são mensurados a partir de amostras de urina e, associado ao PSA, tem se mostrado com sensibilidade de $67 \%$ e especificidade de $83 \%$. Um dos fatores limitantes de seu uso, entretanto, além do custo, é que por ele "passam despercebidos" cânceres mais avançados (CHEETHAM, 2014 \& LOEB et al, 2012).

\section{ESTÁGIOS DO CÂNCER DE PRÓSTATA}

O estágio do câncer de próstata é feito de duas maneiras: clinicamente (avaliação do médico através da consulta e de exames laboratoriais e de imagem) e patologicamente 
(avaliação dos fragmentos da biópsia). O primeiro é baseado no toque retal, PSA, achados de biópsia por agulha fina e imagens radiológicas. O segundo é feito mediante remoção (parcial ou total) de próstata, linfonodo e vesículas seminais com avaliação pelo médico patologista.

A classificação é feita de acordo com o Sistema de Whitmore e Jewett (1956): TNM. $\mathrm{O}$ "T" refere-se a presença de tumor primário e demais características como porcentagem de células cancerosas, meio de descoberta do tumor, entre outros. O "N" refere-se a linfonodos, quanto a presença ou não de extensão linfonodal do tumor. O "M", por fim, refere-se a presença de metástase (COOPERBERG et al, 2013 \& LOEB et al, 2012).

\subsection{Parâmetros Clínicos}

Apesar de o PSA não poder ser usado isoladamente para predição das características do tumor, seus valores aumentados, especialmente acima de $10 \mathrm{ng} / \mathrm{ml}$, são sugestivos de tumores maiores e/ou mais letais. Os outros marcadores tumorais (como isoformas do PSA) também podem ser auxiliares. $O$ toque retal parece ser bastante eficaz na avaliação da extensão tumoral, no entanto, seus resultados são operador-dependente, ou seja, dependem da experiência do médico em realizar tal exame.

A biópsia por agulha fina é realizada e levada ao patologista que realiza a classificação de Gleason já mencionada neste artigo. Uma limitação deste tipo de biópsia é em tumores de pequenas dimensões. Visto que o câncer de próstata comumente realiza metástase óssea, a cintilografia óssea pode ser um exame auxiliar relativamente importante. Entretanto, a cintilografia em pacientes com cânceres menos graves tem um alto índice de falsos-negativos. Atualmente, recomenda-se a cintilografia nos seguintes casos: PSA maior que 20ng/ml, Escore de Gleason de 8 a 10, estágio clínico T3 ou T4 (T3: tumor palpável além da próstata; T4: tumor fixado ou invadindo estrutura adjacentes) ou pacientes sintomáticos (COOPERBERG et al, 2013 \& LOEB et al, 2012).

\subsection{Sintomatologia}

O câncer de próstata é silencioso. Os primeiros estágios não possuem sintomas, daí a importância em realizar a triagem através dos exames de PSA e toque retal. Os sintomas, via de regra, começam a aparecer apenas em estágios mais avançados e são comuns tanto ao câncer quanto à hiperplasia prostática benigna, necessitando, obviamente, de uma avaliação pelo urologista. Entre os sintomas, podemos citar: urinar 
pequeno volume e muitas vezes ao dia (especialmente a noite, obrigando-o a acordar), dificuldade para urinar, dor para urinar e/ou ejacular e presença de sangue na urina ou sêmen (COOPERBERG et al, 2013; EIFLER, 2014 \& LOEB et al, 2012).

\section{ORIENTAÇÕES AO PACIENTE COM DIAGNÓSTICO RECENTE DE CÂNCER DE PRÓSTATA}

Uma das primeiras atitudes tomadas após o diagnóstico de câncer de próstata é o estabelecimento de uma boa relação com seu médico. Vocês entrarão em uma longa jornada que precisará de esforço, comprometimento e confiança de ambos. Por se tratar de uma doença séria, segundas (e terceiras...) opiniões são sempre interessantes, especialmente se o médico tratá-lo de forma extrema - "não é nada" ou "é a pior coisa do mundo". Obviamente, existem exceções.

A decisão de tratamento depende de vários fatores, como idade do paciente, outras doenças, classificação do tumor, estágio, entre outros. Entre as opções, temos cirurgia, radioterapia, terapia hormonal e combinações destas. A quimioterapia não é tão comum. O câncer de próstata, em alguns pacientes, tem um crescimento praticamente "inócuo", ou seja, que não levará o paciente à morte. Em alguns desses casos, pode-se optar por não tratar e só acompanhar o desenvolvimento do tumor ao longo do tempo (SMITH e CROSS, 2014).

\subsection{Muito Baixo Risco}

Pacientes de classificação "muito baixo risco" de acordo com classificações da Associação Europeia de Urologia e Associação Americana de Urologia são os que podem experimentar esse "não tratar". Esse grupo tem poucas chances de que o tumor leve a óbito em um período de 20 anos, levando a considerar a idade do paciente para isso. Caso isso ocorra em pacientes mais jovens, pode-se optar pelo tratamento do grupo de "baixo risco" a seguir.

\subsection{Baixo Risco}

Pacientes de "baixo risco" têm indicação de prostatectomia (cirurgia que remove a próstata) ou de radioterapia, de acordo com outras características. Os outros tratamentos não são recomendados e o "não tratar" até pode ser aplicado, desde que em situações 
especiais e com acompanhamento frequente (ainda não definido pelas autoridades).

\subsection{Risco Intermediário}

Para pacientes com "risco intermediário" a terapia hormonal é uma das primeiras escolhas, no entanto, cirurgia e radioterapia também devem ser opções.

\subsection{Alto Risco}

Para pacientes com classificação "alto risco" a prostatectomia e radioterapia são a primeira escolha. Em pacientes impossibilitados de realizar ambos, pode-se fazer uso da terapia hormonal, porém com acompanhamento frequente (CATALONA et al, 2012; COOPERBERG et al, 2013).

\subsection{Câncer de Próstata Metastático Sintomático}

Pacientes com câncer metastático e com sintomatologia têm terapia hormonal como primeira escolha. Somado a isso, ainda como primeira linha de tratamento, pode-se realizar orquiectomia bilateral (remoção dos testículos) e o uso de medicamentos chamados de agonistas do Hormônio Liberador de Gonadotropinas (GnRH) (COOPERBERG et al, 2013).

\subsection{Câncer de Próstata Metastático Assintomático}

Para pacientes assintomáticos com metástase, a terapia hormonal é escolhida. Alguns autores recomendam que se espere que o paciente desenvolva sintomas antes de iniciar a medicação, realizando enquanto isso um intenso e severo acompanhamento. Ainda não há nada definitivo (COOPERBERG et al, 2013).

\section{CONCLUSÃO}

O câncer de próstata é a segunda neoplasia mais comum no sexo masculino, podendo acometer qualquer grupo étnico e social, mas predominando em homens negros, idosos e com maior nível socioeconômico. Por ser uma doença silenciosa e potencialmente curável, recomenda-se que todos os homens com idade entre 40 e 75 anos sejam 
submetidos a um rastreio através do toque retal anual, ficando a utilização de PSA restritos a alguns casos específicos. O diagnóstico definitivo só pode ser feito a partir da análise da biópsia e o tratamento deve ser guiado a partir desse resultado.

\section{REFERÊNCIAS}

ABOUASSALY, R.; THOMPSON JR, I.M.; PLATZ, E.A.; et al. Epidemiology, Etiology and Prevention of Prostate Cancer. In: KAVOUSSI, L.R.; PARTIN, A.W.; NOVICK, A.; et al. Campbell-Walsh Urology. Filadélfia: Elservier, 2012. 10ªed. P.2704-2725.

BERMAN, D.M.; RODRIGUEZ, R.; VELTRI, R. Development, Molecular Biology and Physiology of the Prostate. In: KAVOUSSI, L.R.; PARTIN, A.W.; NOVICK, A.; et al. Campbell-Walsh Urology. Filadélfia: Elservier, 2012. 10ªed. P.2533-2570.

CATALONA, W.J.; HAN, M. Definitive Therapy for Localized Prostate Cancer: An Overview. In: KAVOUSSI, L.R.; PARTIN, A.W.; NOVICK, A.; et al. Campbell-Walsh Urology. Filadélfia: Elservier, 2012. 10aed. P.2771-2789.

COOPERBERG, M.R.; PRESTI JR, J.C.; SHINOHARA, K.; et al. Neoplasms of the Prostate Gland. In: McANINCH, J.W.; LUE, T.F. Smith e Tanagho's General Urology. Nova lorque: Mc Graw Hill, 2013. 18ª̂ed. P.350-379.

CHEETHAM, P.J. Markers in Prostate Cancer. In: TEWARI, A.K.; WHELAN, P.; GRAHAM, J.D. Prostate Cancer: Diagnosis and Clinical Management. Chichester: Wiley Blackwell, 2014. P.49-71.

DARVES-BORNOZ, A.; PARK, J.; KATZ, A. Prostate Cancer Epidemiology. In: TEWARI, A.K.; WHELAN, P.; GRAHAM, J.D. Prostate Cancer: Diagnosis and Clinical Management. Chichester: Wiley Blackwell, 2014. P.1-15.

EIFLER, J.B. Prostate cancer screening and diagnosis. In: PARSONS, J.K.; EIFLER, J.B.; HAN, M. Handbook of Urology. Chichester: Wiley Blackwell, 2014. P.129-137.

HALL, J. E. Funções Reprodutivas e Hormonais Masculinas (e Função da Glândula Pineal). 
In: HALL, J. E. Guyton \& Hall: Tratado de Fisiologia Médica. Filadélfia: Elsevier, 2011. 12ª̂ed. P.1025-1039.

INSTITUTO NACIONAL DO CÂNCER (INCA). Câncer de Próstata. 2016. Disponível em: http://www2.inca.gov.br/wps/wcm/connect/tiposdecancer/site/home/prostata Acesso em: 25/07/2017.

LOEB, S.; CARTNER, H.B. Early Detection, Diagnosis and Staging of Prostate Cancer. In: KAVOUSSI, L.R.; PARTIN, A.W.; NOVICK, A.; et al. Campbell-Walsh Urology. Filadélfia: Elservier, 2012. 10ªed. P.2763-2769.

MANDAIR, D.; ROSSI, R.E.; PERICLEOUS, M.; et al. Prostate cancer and the influence of dietary factors and supplements: a systematic review. Nutrition \& Metabolism 2014 Jun 16; 11:30. doi: 10.1186/1743-7075-11-30.

MOYER, V.A.; et al. Screening for Prostate Cancer: U.S. Preventive Services Task Force Recommendation Statement. Annals of Internal Medicine, 2012 Jul 17; 157(2):120-34. doi: 10.7326/0003-4819-157-2-201207170-00459.

OXLEY, J. Understanding the Histopathology. In: TEWARI, A.K.; WHELAN, P.; GRAHAM, J.D. Prostate Cancer: Diagnosis and Clinical Management. Chichester: Wiley Blackwell, 2014. P.34-48.

SILVERTHORN, D. U. Reprodução Masculina. In: SILVERTHORN, D. U. Fisiologia Humana: Uma abordagem integrada. Porto Alegre: Artmed, 2010. 5ª ed. P. 838-843.

SMITH, N.J.; CROSS, W.R. Counseling the Patient with Newly Diagnosed Prostate Cancer, Stage by Stage. In: TEWARI, A.K.; WHELAN, P.; GRAHAM, J.D. Prostate Cancer: Diagnosis and Clinical Management. Chichester: Wiley Blackwell, 2014. P.116-135.

SOBREIRO, B.; PASQUALOTTO, F. Câncer de Próstata. Saúde do Homem. 3ª̣ed. Curitiba. 2015.

SOCIEDADE BRASILEIRA DE UROLOGIA. Nota Oficial - Rastreamento do Câncer de 
Próstata. 2015. Disponível em: http://portaldaurologia.org.br/uncategorized/nota-oficialrastreamento-do-cancer-de-prostata/

Acesso em: 25/07/2017.

SOCIEDADE BRASILEIRA DE UROLOGIA. Órgão americano volta atrás em recomendação de rastreamento para o câncer de próstata. 2017. Disponível em: http://portaldaurologia.org.br/noticias-publico/orgao-americano-volta-atras-em-recomendacao -de-rastreamento-para-o-cancer-de-prostata. Acesso em: 25/07/2017.

TRABULSI, E.J.; HALPERN, E.J.; GOMELLA, L.G. Ultrasonography and Biopsy of the Prostate. In: KAVOUSSI, L.R.; PARTIN, A.W.; NOVICK, A.; et al. Campbell-Walsh Urology. Filadélfia: Elservier, 2012. 10ªed. P.2735-2747.

UNITED STATES PREVENTIVE SERVICE TASK FORCE (USPSTF). Prostate Cancer Screening Draft Recommendations. 2017. Disponível em: https://screeningforprostatecancer.org. Acesso em: 25/07/2017. 